\title{
Meshing effects on CFD modelling of atmospheric flow over buildings situated on ground with high terrain
}

\author{
A. Karim, P. Nolan \& A. Qubian \\ Chemical Engineering Department, London South Bank University, UK
}

\begin{abstract}
A Computational Fluid Dynamics (CFD) study was carried out on the wind environment and vehicle pollution dispersion from a newly built by-pass adjacent to the Whatman International site in Maidstone, UK.

The site buildings are sited on ground incorporating an area of high terrain, accordingly they were modelled using Geographical Information System (GIS). The site contains a substantial number of trees of differing species which were extensively surveyed and modeled using a simple 2D momentum sink dependent on the tree Leaf Area Density (LAD).

One of the important factors which has a significant effect on CFD results is the computational mesh. The purpose of this paper is to investigate the effects of using different mesh approaches for both easterly and southerly wind scenarios and the results are compared with that of field measurements taken at the site which include the $\mathrm{CO}$ concentration and wind velocity.

The CFD results showed that the hexahedral mesh delivers a higher level of agreement with field measurements than the tetrahedral dominant mesh and this is mainly due to the higher truncation error in the tetrahedral cell type.

It was also found that the tetrahedral dominant mesh can be significantly improved by applying different numerical solving techniques such as the Nodebased gradient solver.
\end{abstract}

Keywords: CFD, pollution dispersion modelling, grid effect, urban environment.

\section{Introduction}

The Whatman International factory is sited in Maidstone, Kent / UK, the factory consists of a number of buildings which are sited on a ground characterized by 
terrain forming a small valley on either side of the river Medway, whilst a new dual carriageway (A229 bypass) is sited on the upper side of the terrain. The factory manufactures special types of filters which are highly sensitive to any type of air pollutants. The main objective of the study is to investigate the pollutant dispersion and wind environment from the A229 by-pass onto the sensitive manufacturing facility of this factory. Whatman International were seeking to obtain the concentration of certain pollutants at specific sensitive locations in their factory, therefore the pollutant concentration and velocity were measured at those points in the site.

The Computational Fluid Dynamics (CFD) approach was used to simulate the wind environment and to investigate the effects of $\mathrm{CO}$ dispersion produced by the traffic passing through the dual-carriageway on the sensitive manufacturing facility. The preprocessor GAMBIT of the commercial CFD code Fluent [4] was used to design both the geometry and mesh of this site and the Fluent solver is used to solve the Reynolds Averaged Navier Stokes (RANS) equations. However, the footprints and locations of the buildings and land terrain were obtained using GIS and those were used to build the 3D geometry for the CFD simulation, Fig. 1.

From the CFD analysis point of view, one of the main points to be considered is the meshing approach which has significant effects on the prediction and accuracy of the results [1], and this is particularly true of atmospheric flow problems with sometimes very complex geometries (buildings and terrain). Not only is this true of the mesh size but also in the mesh type used (structured / unstructured, hexahedral dominant / tetrahedral dominant etc).

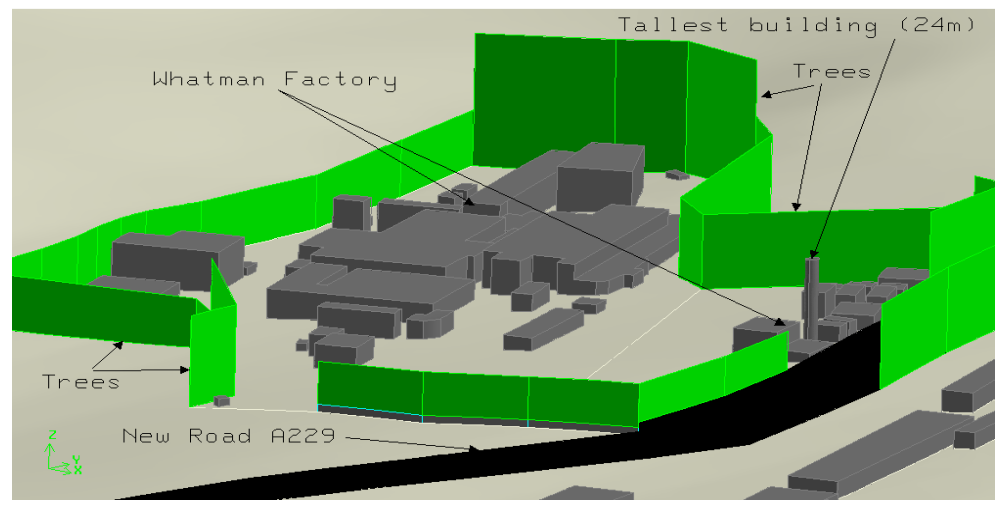

Figure 1: CFD model of the Whatman Intl site and surrounding area.

This paper presents the two main meshing approaches (hexahedral and tetrahedral). The CFD results are compared with that of the field measurements of velocity and $\mathrm{CO}$ concentrations. Accordingly, the mesh approaches and limitations are discussed briefly in the following section, and this is followed by a discussion of the modelling technique, and the paper finishes with the results of specific simulations and their findings. 


\section{Mesh generation}

Generally speaking, the domain of a CFD analysis is usually divided into a number of cells (mesh) in which the RANS equations are solved. However, there are two main types of meshing approaches, structured and unstructured. The structured mesh approach generally ensures stability in convergence, however it is impractical for the real atmospheric flow cases [2,3]. On the other hand, the unstructured mesh approach would have higher numerical errors which significantly vary depending on the type of the cells (e.g hexahedral or tetrahedral) and the degrees of the cell quality.

It is worth discussing briefly the difference between the hexahedral cell shape and the tetrahedral cell shape with regards to the solution accuracy. The face value of the cells used in the Fluent software to calculate the various flow variables is dependent on the interpolation method used and that involves different schemes to calculate values from cell to cell.

For example the cell face value for any given variable $\phi_{f}$ can be determined using equation (1) (typical interpolation equation), and this is dependent on the cell centroid value $\phi_{c}$, cell gradient $\nabla \phi$, and the geometrical features of the cell as defined in Fig. 2. Thus if we take two near wall adjacent cells of hexahedral and tetrahedral shape shown in Fig. 2 (here the flow is assumed to be parallel to the wall), with hexahedral cells the face is on a vertical angle whilst the tetrahedral cells are inclined and this gives rise to a non-zero gradient. This very well known phenomenon results in a higher truncation error.

$$
\phi_{f}=\phi_{c}+\delta r(\nabla \phi)_{c}+\left(|\delta r|^{2}\right)
$$

However if the flow is not aligned to the cells (not parallel), the hexahedral mesh looses its "edge" on the tetrahedral mesh scheme. For atmospheric flow problems typically the flow can be easily and naturally aligned with the hexahedral cell arrangement.

Hexahedral face

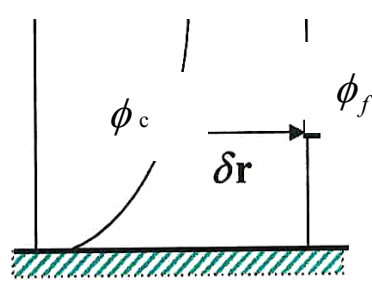

$$
\left[\delta r(\nabla \phi)_{c}\right]=0
$$

Tetrahedral face
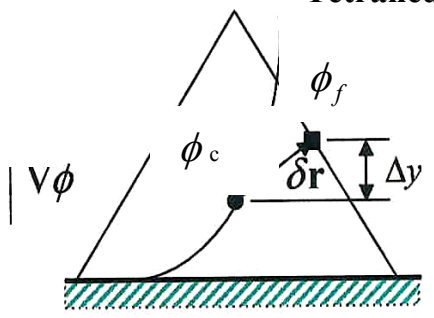

$\left[\delta r(\nabla \phi)_{c}\right] \neq 0$

Figure 2: Diagram illustrating the effect of mesh shape on the simulation accuracy. 
For the present CFD work, two mesh schemes were used; i) The Hexahedral Core (HC) type [4] which is a tetrahedral dominant mesh ii) The second mesh type used is the wholly hexahedral type, Hexahedral-Cooper (Cooper). The results of both approaches will be discussed in order to highlight their level of agreement with field measurements.

Due to the requirement of an orthogonal cell in the near wall area studies have shown improved simulations using prism shapes in the tetrahedral mesh [5]. However the present work has sought to compare between the tetrahedral mesh and hexahedral mesh primarily.

For the Hex-Cooper mesh, the maximum skewness was approximately 0.9 whilst the maximum aspect ratio was 200. The Cooper mesh contained 2.7 million cells in total.

The HC mesh, on the other hand, contained a maximum skewness of 0.95 , however the aspect ratio is fairly low throughout the domain. The HC mesh was about 2.6 million cells.

\section{Modelling technique}

For the CFD modelling procedure, there are many important factors which should be carefully chosen for solving the RANS equations. Among them are the turbulence model, the domain boundary conditions and the calculation of the cell gradient values. Brief discussions on these issues are given below.

For the domain boundary condition, the side flow inlet is modelled using approximated profiles suggested by Richards and Hoxey [6]. The profiles would be calculated using meteorological data taken at the nearby East Malling station. The aforementioned profiles are commonly used in literature and seem to give suitable approximations to the flow variation, they include the wind speed, turbulent kinetic energy and dissipation [7]. All other domain boundaries are specified as symmetry whilst the outlet is set as an outflow boundary [8].

A line of substantially tall trees almost entirely surrounds the Whatman site, these were modelled as 2D momentum sinks, with turbulence generation inside the canopy ignored. This was based on data from the UK Forestry Commission on Leaf area Density (LAD).

For modelling turbulence the standard k- $\varepsilon$ modified by Detering and Etling [9] is used, with standard wall functions. The vehicle pollutant from the road is released as an area emission with the turbulence generated by vehicles ignored.

For the cell gradient values; the Fluent code provides three methods for calculating the gradient values of each individual cell from the surrounding cells, Green-Gauss cell-based, Green-Gauss Node-based and Least squares cell-based, for more details see [4].

However, the CFD results could be significantly improved by using the Nodebased method, this is particularly true for the arbitrary shape arrangement of the cells which are associated with the tetrahedral meshing scheme $[4,10]$. The Node-based method calculates the node weighted average for all surrounding cells which share the same node. This is especially critical for the tetrahedral mesh scheme because significantly more cells share any single node than the 
hexahedral arrangement, thus the numerical errors become naturally higher. However, the iteration time would be significantly increased, and this may be more severe (double or triple) when tackling more complex problems, e.g reactions.

Accordingly, in order to improve the result of the tetrahedral based mesh without seeking higher numerical schemes or more complex turbulence models the Node-based gradient method is utilized.

\section{Results and discussions}

In order to validate the CFD models against the results of the experimental work, the two dominant wind directions of both east and south were investigated. The CFD prediction involved the use of two meshing schemes, first the Cooper scheme which is an entirely hexahedral mesh, whilst, the second one, $\mathrm{HC}$ is a tetrahedral dominant mesh, and the main features of those schemes are presented below.

\subsection{Wake flow behind the buildings}

Generally speaking, for the dispersion of the $\mathrm{CO}$ pollutant, the wake flow behind the buildings represents one of the important fluid features which would participate in the changes of pollutant concentration locally and also in channeling and directing the flow movement downstream of the buildings.

For the Cooper meshing approach, the predictions of flow movement within the wake behind the buildings were predicted with some considerable clarity and accuracy in their sizes, flow separation and flow directions.

For the $\mathrm{HC}$ tetrahedral approach the wake formation is much less clear and the wake region is larger in size and this is typical of all the buildings in the domain. Another point worth mentioning here, is the flow reattachment point downwind of the buildings are further downstream in the $\mathrm{HC}$ mesh as compared to the Cooper scheme, and this is shown in Figs. 3 and 4 around a typical site building.

However, the differences in results can be explained as follows; for the HC meshing approach, despite a higher number of cells filling the wake region behind the building which in fact slightly exceeded that of the Cooper scheme, but due to the random structure arrangement of the tetrahedral cells, which mean that the cell centers are in different non-equidistant locations in relation to neighboring cells, the consequences of that, are the solver will interpolate values carrying a higher numerical error which is exponentially expanded downstream, Figs 5 and 6.

The hexahedral shape at the wall is known to give improved predictions due to the better resolution of turbulent energy normal to the wall [11]. Accordingly the $\mathrm{HC}$ mesh results showed that the flow near the ground terrain region is characterized by small fluctuations (bumps) in the velocity plot, whilst the Cooper mesh did not have these inconsistencies. 
The features discussed above had a significant effect on the $\mathrm{CO}$ concentration as shown in Figs. 7 and 8. The CO concentration for the HC mesh is highly irregular with sporadic high concentration points.

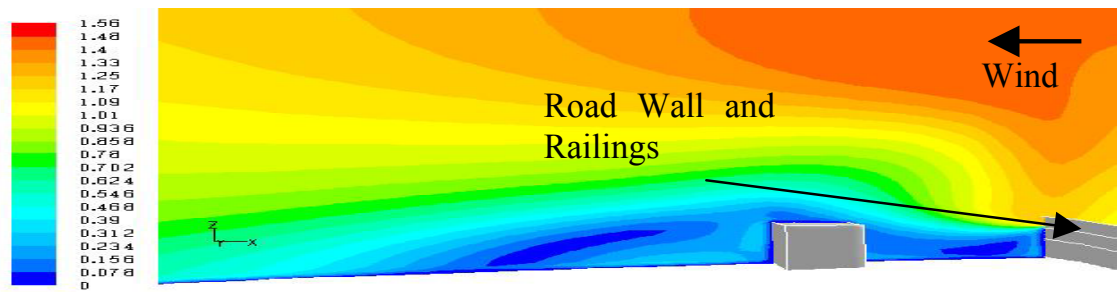

Figure 3: Velocity contours in vertical plane (m/s)-East wind, (Cooper).
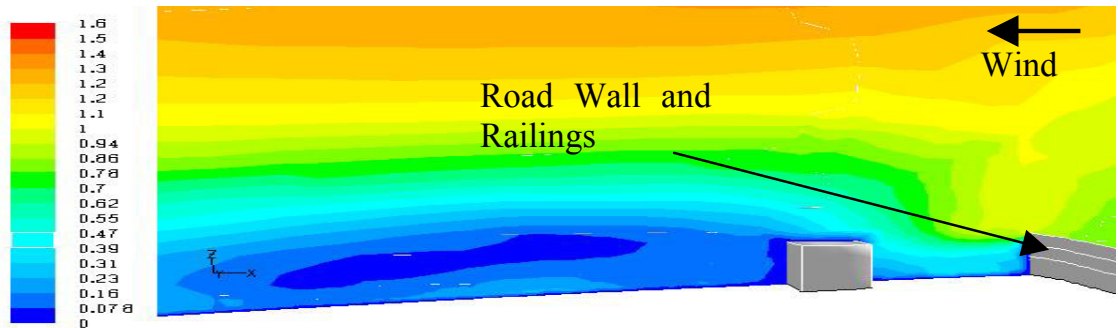

Figure 4: Velocity contours in vertical plane (m/s)-East wind, (HC).

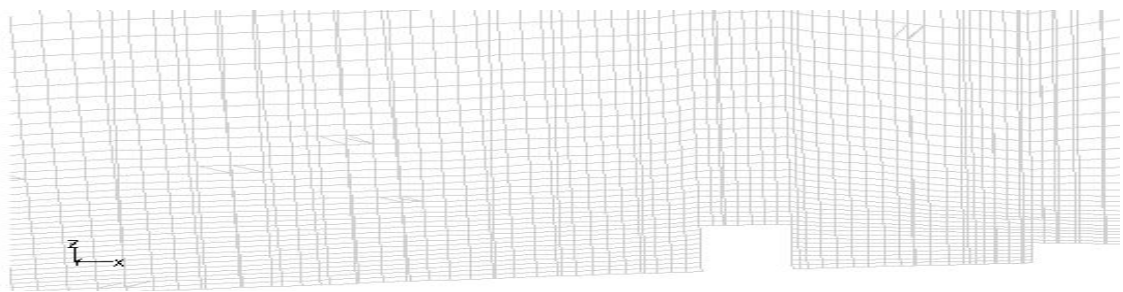

Figure 5: $\quad$ Mesh in vertical plane surrounding typical site building, (Cooper).

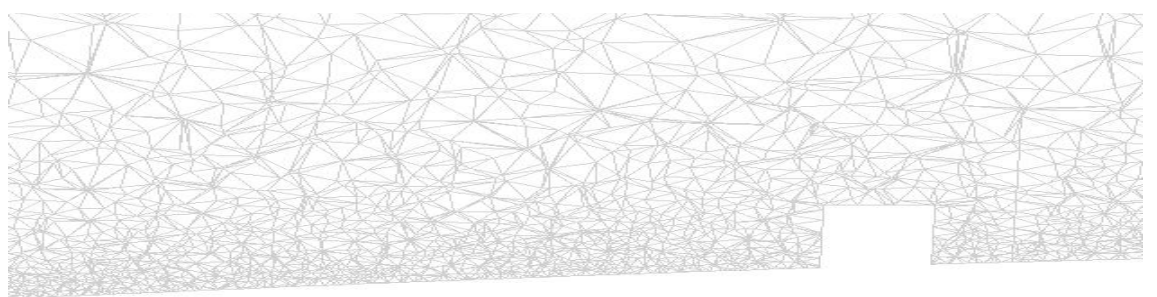

Figure 6: $\quad$ Mesh in vertical plane surrounding typical site building, (HC). 

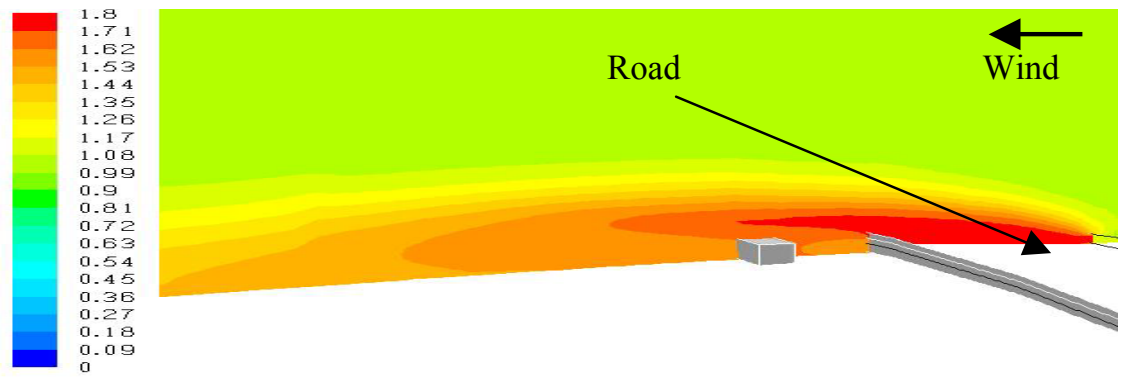

Figure 7: $\quad \mathrm{CO}$ concentration contours in vertical plane (PPM)-East wind, (Cooper).

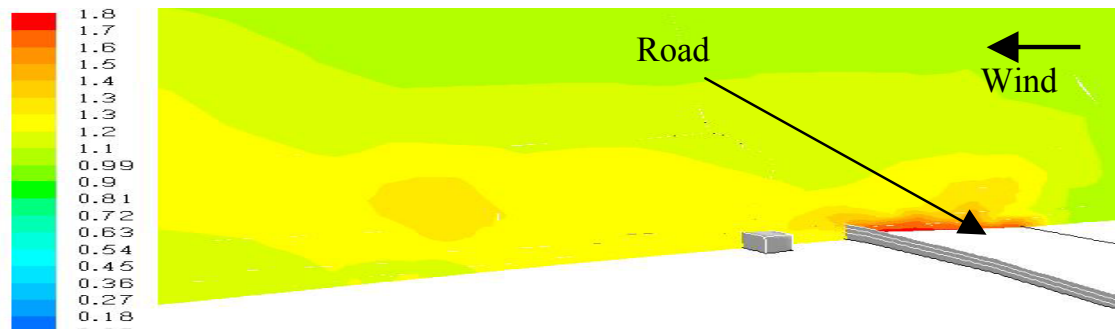

Figure 8: $\quad \mathrm{CO}$ concentration contours in vertical plane (PPM)-East wind, (HC).

\subsection{Wind velocity and Carbon Monoxide concentration}

The measurements of $\mathrm{CO}$ concentration were carried out at a location $10 \mathrm{~m}$ away from the road, whilst the wind velocity measurements were carried out $60 \mathrm{~m}$ away from the road. Generally speaking, the CFD predicted results are in good agreement with those measured, see Table 1 .

For the CFD results, the Cooper mesh scheme produces better overall agreement with that of the measurements and the discrepancies of both $\mathrm{CO}$ concentration and wind velocity were $12.5 \%$ and $3 \%$ respectively for the east wind and $16 \%$ and $1 \%$ for the south wind.

Given the drastic differences between the meshing schemes in predicting wake size behind buildings and the boundary layer development, accordingly, in using the $\mathrm{HC}$ mesh scheme, the discrepancies of both $\mathrm{CO}$ prediction and wind velocity were $20 \%$ and $13 \%$ respectively for the east wind, whilst it is slightly higher for the southerly wind, $32 \%$ and $26 \%$ respectively.

Those high discrepancies which are usually associated with the tetrahedral meshing scheme would be significantly attributed to the numerical and truncation errors. As a result of that the distributions within the complex flow regions such as the wakes and boundary layer formation on the terrain would be significantly changed. However, the predicted velocity or $\mathrm{CO}$ obtained for the 
Table 1: Summary of the CFD simulations as compared to the field measurements.

\begin{tabular}{|c|c|c|c|c|c|c|c|}
\hline $\begin{array}{l}\text { Wind } \\
\text { Direction }\end{array}$ & Mesh Type & $\begin{array}{l}\text { Velocity } \\
\text { measured } \\
(\mathrm{m} / \mathrm{s})\end{array}$ & $\begin{array}{l}\text { Velocity } \\
\text { CFD } \\
\text { (m/s) }\end{array}$ & $\begin{array}{l}\text { Velocity } \\
\text { Error } \\
(\%) \\
\end{array}$ & $\begin{array}{l}\mathrm{CO} \\
\text { measured } \\
\text { (PPM) }\end{array}$ & $\begin{array}{c}\text { CO } \\
\text { CFD } \\
\text { (PPM) }\end{array}$ & $\begin{array}{l}\text { CO } \\
\text { Error } \\
(\%) \\
\%\end{array}$ \\
\hline East & $\begin{array}{l}\text { Hexahedral } \\
\text { (Cooper) }\end{array}$ & 0.70 & 0.72 & 3 & 1.69 & 1.48 & 12.5 \\
\hline East & $\begin{array}{l}\text { Tetrahedral } \\
(\mathrm{HC})\end{array}$ & 0.70 & 0.60 & 13 & 1.69 & 2.03 & 20 \\
\hline East & $\begin{array}{l}\text { Tetrahedral } \\
\text { (HC-Node } \\
\text { based) }\end{array}$ & 0.70 & 0.73 & 4 & 1.69 & 1.98 & 17 \\
\hline South & $\begin{array}{l}\text { Hexahedral } \\
\text { (Cooper) }\end{array}$ & 1.00 & 1.01 & 1 & 1.06 & 1.23 & 16 \\
\hline South & $\begin{array}{l}\text { Tetrahedral } \\
(\mathrm{HC})\end{array}$ & 1.00 & 1.26 & 26 & 1.06 & 1.41 & 32 \\
\hline South & $\begin{array}{l}\text { Tetrahedral } \\
\text { (HC-Node } \\
\text { based) }\end{array}$ & 1.00 & 1.1 & 10 & 1.06 & 1.37 & 28 \\
\hline
\end{tabular}

free-stream regions of the domain have good agreements with field measurements.

The highest discrepancies of both wind velocity and $\mathrm{CO}$ concentrations associated with the southern wind scenario can be related to the fact that the unsettled and unsteady flow phenomena such as the wakes, flow separation and recirculation become more significant within the reference points which are used in the measurements, accordingly, the predicted CFD results would have increased numerical and truncation errors.

On the other hand, for the $\mathrm{HC}$ tetrahedral mesh case, the CFD results were improved significantly by using the Node-based gradient method comparing to that of Cell-based method (default Fluent option), Table 1. The predicted CO and velocity at the measurement points become comparable to that obtained by the Cooper mesh scheme

\section{Conclusion}

Two CFD models for the Whatman international site in Kent, UK were designed and solved using fully hexahedral Cooper mesh and the HC tetrahedral dominant mesh with the same flow conditions for the easterly and southerly wind scenarios. Both models were solved with the same turbulence model and numerical schemes and with almost the same number of meshing cells.

Both schemes generally predicted the same overall flow characteristics; however the $\mathrm{HC}$ scheme compared to the Cooper scheme produced irregular plots of $\mathrm{CO}$ and velocity in the wake regions and along the ground which has a varying terrain, as well as differences in the prediction of the wake sizes behind buildings.

When compared to the field measurements, the $\mathrm{HC}$ mesh predicted lower localized velocities which led to over-prediction of the $\mathrm{CO}$ concentration. The $\mathrm{HC}$ mesh gave discrepancies of up to $16 \%$ in wind velocity and $25 \%$ in $\mathrm{CO}$ 
concentration, whilst the Cooper scheme produced discrepancies of $3 \%$ in the velocity and $16 \%$ in the $\mathrm{CO}$ concentration.

The results strongly suggested that the $\mathrm{HC}$ scheme, in non-complex flow regions of the domain (complex being areas of separation or recirculation regions) produced velocity and $\mathrm{CO}$ predictions which were quite reasonable. However the performance of the HC mesh is greatly influenced by the complex flow phenomenon which occurred for the south wind scenario and that produced much higher discrepancies, whilst the Cooper scheme was largely unaffected

Furthermore it was found that the $\mathrm{HC}$ mesh numerical errors could be greatly reduced by running the solver using the Node-based method for calculating the cells' gradient values instead of using the Cell-based method which is the typical method employed in most commercial codes.

\section{Acknowledgements}

The authors would like to thank Dr. Olga Grant of the East Malling Research Centre, and Dr. Rona Pitman and Chris Peachey of UK Forestry Commission.

\section{References}

[1] Cowan, I. R., Castro, I.P., Robins, A.G. Numerical considerations for simulations of flow and dispersion around buildings. Journal of Wind Engineering and Industrial Aerodynamics 67 \& 68 pp. 535-545, 1997.

[2] Huber, A., Freeman, M., Spencer, R., Schwarz. W., Bell, B., Kuehlert, K. Development and applications of CFD simulations supporting urban air quality and homeland security, AMS sixth symposium on the urban environment, Atlanta, U.S, 2006.

[3] Kim, S., and Boysan, F. Application of CFD to environmental flows, Journal of Wind Engineering and Industrial Aerodynamics, 81 pp. 145$158,1999$.

[4] Fluent user guide. Fluent. Inc 2006

[5] Fothergill, C.E., Roberts, P.T., Packwood, A.R. Flow and dispersion around storage tanks. A comparison between numerical and wind tunnel simulations. Wind and structures, Vol. 5, No. 2-4 pp. 89-100, 2002

[6] Richards, P.J. and Hoxey, R. Appropriate boundary conditions for computational wind engineering models using the k-e model, Journal of Wind Engineering and Industrial Aerodynamics, 46, 47, pp. 145-153, 1993.

[7] Hargreaves, D.M. and Wright, N. G. On the use of the k- $\varepsilon$ model in commercial CFD software to model the neutral atmospheric boundary layer. Journal of Wind Engineering and Industrial Aerodynamics, doi: 10.1016/j.weia.2006.06.002, 2006

[8] Franke, J. et al Recommendations on the use of CFD in predicting pedestrian wind environment. COST Action C14 "Impact of wind and storms on city life and built environment" Working Group 2-CFD techniques, 2004 
[9] Detering, H.W. and Etling, D. Application of the E- $\varepsilon$ Turbulence Model to the Atmospheric Boundary Layer. Boundary Layer Meteorology $\mathbf{3 3}$ pp. 113-133, 1985

[10] Ferziger, J. H. and Peric, M. Computational Methods for Fluid Dynamics, Springer - Verlag: New York, 2002.

[11] Fothergill, C. E., Roberts, P.T., Packwood, A.R. Flow and dispersion around storage tanks. A comparison between numerical and wind tunnel simulations. Wind and Structures, Vol. 5, 2 4 pp. 89-100, 2002. 